Annals of Pure and Applied Mathematics

Vol. 19, No. 1, 2019, 21-35

ISSN: 2279-087X (P), 2279-0888(online)

Published on 29 January 2019

www.researchmathsci.org

DOI: http://dx.doi.org/10.22457/apam.591v19n1a4

Annals of

Pure and Applied

Mathematics

\title{
Mathematical Modelling on the Effects of Drug Abuse to the Societies
}

\author{
Fikiri Lucas Matonya $^{1}$ and Dmitry Kuznetsov ${ }^{2}$ \\ Department of Applied Mathematics \\ The Nelson Mandela African Institute of Science and Technology \\ Arusha, Tanzania \\ ${ }^{2}$ E-mail: dmitry.kuznetsov@nm-aist.ac.tz \\ ${ }^{1}$ Corresponding author. E-mail: fikiri.lucas@gmail.com \\ Received 16 December 2018; accepted 15 January 2019
}

\begin{abstract}
The global burden of death and disability attributable to drug users, remains a significant threat to public health for both developed and developing countries. This paper present a new mathematical modelling framework to investigate the effects of drug use in the community. In our model the transmission process is considered as a social contact process between the susceptible individuals and drug users based on the epidemiology principles. An epidemic threshold value, $R_{0}$, is proposed for the drug-using career. Sensitivity analysis is performed on $R_{0}$, and it is then used to examine the stability of the system. A condition under which a backward bifurcation may exist is found, as are conditions that permit the existence of one or more endemic equilibria. A key result arising from this model is that prevention is indeed better than cure.
\end{abstract}

Keywords:Drugs, drug abuse, drug abuse number, sensitivity analysis

AMS Mathematics Subject Classification (2010): 96M10

\section{Introduction}

Substance/drug abuse can be viewed as excess in or dependence on a substance, drug or other chemical foremost to effects that are injurious to the individual's physical, social, psychological and mental health and or the welfare of others. Most of the substances abused are psychoactive substances that lead to addiction syndrome when used.

Definition 1.1. Dependence is defined as, "a cluster of cognitive, behavioural, and physiological symptoms indicating that the individual continues the use of the substance despite significant substance abuse related problems" [1].

Substances abused include both legal (not prohibited by law) and criminal (prohibited by law) drugs. They can be classified into different classes depending on the level of abuse and the interest of the classifying organization. In the case of South Africa [2] substances abused have been classified into those which are heavily abused, those temperately abused and those that are less frequently abused. The heavily abused substances include; alcohol (in all its forms), dagga (cannabis), cigarettes, dagga and mandrax combined, even though sometimes mandrax (methaqualone) is used on its own. 


\section{Fikiri Lucas Matonyaand Dmitry Kuznetsov}

Others in the same category include prescription drugs such as slimming tablets, tranquillizers and cough mixtures. Moderately abused substances include; cocaine (cocaine powder), crack- cocaine, heroin,speed, lysergic acid diethylamide (LSD), hashish, and Ecstasy MDMA. Explicit information about health effects, behavior, law and treatment program of Methamphetamine is provided in [3]. The least abused substances include; opium, Rohypnol, ketamine and wellconal.In the USA, different bodies have classified drugs and the drug abuse disorders into different classes.

The Diagnostic and Statistical Manual of Mental Disorders (DSM-IV) divided the drugs and related disorders into 13 classes based on the drugs abused such as alcohol, sedatives, hypnotic or anxiolytic drugs, amphetamines, cocaine, caffeine, cannabis, hallucinogens (such as LSD) nicotine, opioid, phencyclidine (phenylcyclohexylpiperidine PCP) . Other bodies classify drugs as follows; the Diagnostic and Statistical Manual of the American Psychiatric Association, 13 categories, U.S DEA's and Coast Guard Scheme, 6 categories, Julien Biomedical-Type Scheme into 9 categories, Sussman and Ames Health Promotion Subjective-Behaviour Scheme into 8 categories (S. Sussman and S. L. Ames. Drug Abuse: concepts, prevention and cessations. Cambridge University Press, 2008). We note that, even though the classification of substances may differ depending on the classifying body, the substances abused and the resulting effects are the same.

Drug addiction is a brain disease with well recognized cognitive, behavioural and physiological characteristics that contribute to compulsive and continued use of drugs despite the harmful consequences. The fact that recovery from drug addiction takes time, and addicts are at a high risk of relapse, effective rehabilitation programmes are not only required but also must last long enough to produce stable behavioural change and maintain abstinence over time. Scientist have also found that chronic drug abuse alters the brain's anatomy and chemistry and that these changes last for months or years after the individual has stopped using drugs. The major example was published by Wolkow et al.[4] on the lasting changes in the brain and the heart caused by addiction.

The justification of the perplexing and continued abuse of drugs spans basic neurobiological,physiological, social and environmental factors. According to [4], repeated use of drugs changes how the brain functions, and affects the natural inhibition and reward centres of the brain. As a consequence, this results in transition from voluntary to adverse social, health or legal consequences. Although relapse may be largely due to withdraw symptoms and stress, craving for drugs may also be as a result of "spontaneous recovery" triggered by contact with people, places, and things associated with prior drug use.

Substance abuse is associated with many dangers including the less well known such as holoprosencephaly (HPE) in fatal alcohol syndrome and leukoencephalopathy which is associated with inhaled heroin [5]. In this thesis, we detail some of the effects of drugs on the brain, some effects on the neuronal pathways and the resulting damage that can lead to disruption of cognitive and motor functions. The challenges are briefly organized in accordance with specific drugs and we concentrate more on the highly prevalent drugs including injectable drugs. The drugs have different modes of administration which include smoking, snorting (snuffing), oral ingestion and others are intravenously administered. 
Mathematical Modelling on the Effects of Drug Abuse to the Societies

The mode of administration determines the rate at which optimal levels of the drug in blood are reached and the many harms including Chronic Obstructive Pulmonary Disease (COPD), Pulmonary edema, pulmonary hypertension, associated with abusing drugs [6]. The doctrine of types and nature of drug use involves recreational settings, society, family life experiences and psychiatric disorders.

\section{Materials and methods}

\subsection{Model development}

In our model we examines the dynamics of population of drug users in a heterogeneous population. The population is stratified into four classes: those at risk of using drugs(susceptible) denoted by S, lighter users L, Heavy users $\mathrm{H}$ and drug users on treatment $\mathrm{T}$,. The total variable population size at any time $\mathrm{t}$ is given by

$$
N(t)=S(t)+L(t)+H(t)+T(t)
$$

We undertake that the individuals in each compartment are indistinguishable and there isregular mixing. The model assumes that individuals join the susceptible population at a rate $\pi$ through births and immigration. Susceptible individuals are initiated into drug usefollowing interaction with individuals using drugs. We thus assume an initiation function that is analogous to the force of infection for epidemic models. In this case, the per capita contact rate $\beta$ is a product of the effective number of contactsc, between drug users and the susceptible population, and the probability $\hat{\beta}$, that a contact results in initiation into drug use so that $\beta=c \hat{\beta}$.

The parameters $\eta_{1}$ and $\eta_{2}$ amount the relative ability to initiate new drug users for heavy users and those in treatment respectively when compared to light users. Assuming that the rate at which heavy users and those in treatment recruit initiates is lower than that of light users, wehave $0<\eta_{1}, \eta_{2}<1$. This is due to the fact that problematic drug use is associated withmorbidity and this serves as "negative" advertisement.

The total amount relevant contacts gives the initiation function,

$$
\Lambda=\beta\left(\frac{L+\eta_{1} H+\eta_{2} T}{N}\right)
$$

We undertake that the rate at which heavy users and those in treatment recruit initiates is lesser than that of light users, we have $0<\eta_{1}, \eta_{2}<1$.

Upon contamination, a susceptible individual move into the compartment of light users. The light use phase, represents initial phase of drug abuse and individuals can either stop, die or move to heavy drug use. It is at this stage that our model differs from a number of models on substance abuse. We say that this approach is demonstrative of drug use cycles. Heavy drug users can either revert to light drug use, die, join rehabilitation programmes or they are removed due to drug use related problems. Removal due to substance abuse related problems include incarceration and deaths directly caused by the use of drugs. This is of particular importance when one considers the fact that drug abuse often damages judgement for drivers, increases the risk of contracting killer diseases such as HIV and often leads to forceful crimes as addicts search for money to buy drug doses. Once in rehabilitation individuals can either have a 


\section{Fikiri Lucas Matonyaand Dmitry Kuznetsov}

relapse to hard drug use, quit permanently, die or they are also removed due to drug use related problems.

\subsection{Model equations}

The ordinary differential equations that represent the compartmental models are

$$
\begin{gathered}
\frac{d S}{d t}=\pi-\Lambda S-\mu S+\gamma_{1} L \\
\frac{d L}{d t}=\Lambda S+\gamma_{2} H-Q_{1} L \\
\frac{d H}{d t}=\sigma L+\gamma_{3} T-Q_{2} H \\
\frac{d T}{d t}=\rho H-Q_{3} T
\end{gathered}
$$

where

$Q_{1}=\left(\mu+\sigma+\gamma_{1}\right)$,

$Q_{2}=\left(\mu+\rho+\gamma_{2}+\delta_{1}\right)$,

$Q_{3}=\left(\mu+\gamma_{3}+K+\delta_{2}\right)$.

All the model parameters are positive and the initial conditions of the model system 1 are given by

$$
S(0)=S_{0}>0, L(0)=L_{0} \geq 0, H(0)=H_{0} \geq 0 \text { and } T(0)=T_{0} \geq 0 .
$$

Since the model monitors changes in the human population, the variables and the parameters are assumed to be positive for all $t \geq 0$.

\subsection{Basic properties of the model}

\subsubsection{Positivity of solutions of the model}

We now consider the positivity of solutions of the system of equations (1). We prove that all the state variable remains non-negative and the solutions of the system (1) with positive initial conditions will remain positive for all $t>0$. We thus state the following theorem.

Theorem 2.4. Given that the initial conditions of system(1) are $S_{0}>0, L_{0}>0, H_{0}>$ 0 and $T_{0}>0$, the solutions of $S(t), L(t), H(t)$ and $T(t)$ are non-negative for all $t>0$.

Proof: Assume that

$$
\hat{t}=\sup \{t>0: S>0, L>0, H>0, T>0\} \in[0, t]
$$

Thus, $\hat{t}>0$, and it follows directly from the first equation of the system (3) that

$$
\frac{d C}{d t} \geq \boldsymbol{\Pi}-(\lambda+\mu) S
$$

We use the integrating factor method to solve the inequality (4). The resulting inequality can be written as,

$$
\frac{d S}{d t}+(\lambda+\mu) \geq \Pi
$$


Mathematical Modelling on the Effects of Drug Abuse to the Societies

It can be shown that

$$
S(\hat{t}) \geq S(0) e^{-\left(\mu \hat{t}+\int_{0}^{\hat{t}} \lambda(s) d s\right)}+e^{-\omega \hat{t}+\int_{0}^{\hat{t}} \lambda(s) d s}\left[\int_{0}^{\hat{t}} \Pi e^{\left(\mu \hat{t}+\int_{0}^{\hat{t}} \lambda(s) d s\right)} d t\right],>0, \forall \hat{t}>0
$$

Proof: From the second equation of (1)

$$
\begin{gathered}
\frac{d L}{d t}=\Lambda S+\gamma H-\left(\mu+\sigma+\gamma_{1}\right) L \\
\geq-\left(\left(\mu+\sigma+\gamma_{1}\right) L\right. \\
\Rightarrow \geq L_{0} e^{-\left(\mu+\sigma+\gamma_{1}\right) t>0 .}
\end{gathered}
$$

Similarly, it can be shown that $H(t)>0$ and $T(t)>0$ for all $t>0$, and this complete the proof.

\subsection{Boundedness of the state space variable}

[7] In human population models, the objective is to obtain non-negative solutions. The analysis of system (3) therefore be analyzed in region Tof biological interest. We have the following Lemma on the region that system (1) is restricted to Lemma.

The feasible region $\mathrm{D}$ defined by

With initial conditions

$$
\mathrm{D}=\left\{(S(t), L(t), H(t), T(t)) \in R_{+}^{4} \mid 0 \leq N \leq \frac{\Pi}{\mu}\right\},
$$

$$
C_{0} \geq 0, \quad D_{0} \geq 0, S_{0} \geq 0 \text { and } R_{0} \geq 0 .
$$

is positively invariant and attracting with respect to system (1) for all $t>0$.

Proof: The population in is model is clearly not constant. Therefore the evolution equation for change in the population is given by

$$
\begin{gathered}
\frac{d N}{d t}=\Pi-\mu N-\delta_{1} H-\left(K+\delta_{2}\right) T \\
\leq \Pi-\mu N
\end{gathered}
$$

It can be easily be shown that

$$
N \leq \frac{\Pi}{\mu}+\left(N_{0}-\frac{\Pi}{\mu}\right) e^{-\mu t} \text { where } N(0)=N_{0} .
$$

From (3), We observe that as $t \rightarrow \infty, N(t) \rightarrow \frac{\Pi}{\mu}$ implying that $\lim _{t \rightarrow \infty} N(t)=\frac{\Pi}{\mu}$. Clearly, $\frac{\Pi}{\mu}$ is the upper bound of $\mathrm{N}$. On the other hand, if $N_{0}>\frac{\Pi}{\mu}$, then $\mathrm{N}$ will decrease to $\frac{\Pi}{\mu}$ as $t \rightarrow \infty$.

This means that if $N_{0}>\frac{\Pi}{\mu}$, then the solution $(S(t), L(t), H(t), T(t))$ enters $\mathcal{D}$ or approaches it asymptotically. Therefore, $D$ is positively invariant under the flow induced by system (3). Thus, in $\mathcal{D}$ the model (3) is well-posed. Hence, it is sufficient to study the dynamics of the nodel in D.

\section{Model analysis}

\subsection{The drug-free equilibrium and the basic reproduction number}

The central concept in analyzing and quantifying the transmission of the infection is the 


\section{Fikiri Lucas Matonyaand Dmitry Kuznetsov}

reproduction ratio $R_{0}$. As defined by [8], the reproduction number is "the number of minor infections produced by a single infective individual in an entirely susceptible population".

We assume that the drug free steady state exists and it is locally asymptotically stable.By this postulation if, $\mathbb{E}_{0}$ denotes the drug free equilibrium of the system then $\mathcal{F}(x)$ is set to zeros and all eigenvalues of $D f\left(x_{0}\right)$ have negative real parts.

We find the model basic reproduction number using the next generation matrix methoddescribed in [9]. The infective classes involved are $L, H, T$. We decompose the rates of change $(d L / d t, d H / d t, d T / d t)$ of the infective classes in terms of two matrices $f_{i}$ and $v_{i}$ where, $f_{i}$ represents new infections and $v_{i}$ represents transitions. Then we evaluate,

$$
F=\left[\frac{\partial f_{i}}{\partial x_{j}}\left(x_{0}\right)\right] \text { and } V=\left[\frac{\partial v_{i}}{\partial x_{j}}\left(x_{0}\right)\right]
$$

$x_{0}$ is the drug free equilibrium. $F$ is non-negative and $V$ is non-singular. Therefore,

$$
f_{i}=\left(\begin{array}{c}
\Lambda S \\
0 \\
0
\end{array}\right) \quad \text { and } \quad F=\left(\begin{array}{ccc}
\beta & \eta_{1} \beta & \eta_{2} \beta \\
0 & 0 & 0 \\
0 & 0 & 0
\end{array}\right)
$$

In the same way the transition matrix for the model is given by

$$
v_{i}=\left(\begin{array}{c}
-\gamma_{2} H+Q_{1} L \\
-\sigma L-\gamma_{3} T+Q_{2} H \\
-\rho H+Q_{3} H
\end{array}\right) \text { and } V=\left(\begin{array}{ccc}
Q_{1} & -\gamma_{2} & 0 \\
-\sigma & Q_{2} & -\gamma_{3} \\
0 & -\rho & Q_{3}
\end{array}\right)
$$

where $Q_{1}=\left(\mu+\sigma+\gamma_{1}\right), Q_{2}=\left(\mu+\gamma_{2}+\partial_{1}+\rho\right)$ and $Q_{3}=\left(\mu+k+\partial_{2}+\gamma_{3}\right)$.

The basic reproduction number is given as the spectral radius of the next generation matrix

$$
R_{0}=\rho\left(F V^{-1}\right)=R_{01}+R_{02}+R_{03},
$$

The threshold number (reproduction number) is the number of minor initiations into drug use, when a drug user is introduced in a purely susceptible population.We calculate the threshold using the next generation matrix method indicated in (Van den Driessche and Watmough, 2002). Therefore, we have $R_{0}=R_{01}+R_{02}+R_{03}$, Such that

$$
\begin{gathered}
R_{01}=\left(\frac{\beta}{Q_{1}}\right)\left(\frac{1-\Phi_{1}}{1-\left(\Phi_{1}+\Phi_{2}\right)}\right) \\
R_{02}=\left(\frac{\eta_{1} \beta}{Q_{2}}\right)\left(\frac{\sigma}{Q_{1}}\right)\left(\frac{1}{1-\left(\Phi_{1}+\Phi_{2}\right)}\right) \\
R_{03}=\left(\frac{\eta_{2} \beta}{Q_{3}}\right)\left(\frac{\rho}{Q_{2}}\right)\left(\frac{\sigma}{Q_{1}}\right)\left(\frac{1}{1-\left(\Phi_{1}+\Phi_{2}\right)}\right)
\end{gathered}
$$

where, $\Phi_{1}=\frac{\rho \gamma_{3}}{Q_{2} Q_{3}}$ and $\Phi_{2}=\frac{\gamma_{2} \sigma}{Q_{2} Q_{1}}$

$\Phi_{2}$ is the possibility of moving from one of $\mathrm{L}$ and $\mathrm{H}$ to the other and back again.

$\Phi_{1}$ is the possibility of moving from one of $\mathrm{H}$ and $\mathrm{T}$ to the other and direct back.

Eachtime a light user enters a heavy using class, the expected number of new initiation is $\left(\frac{\eta_{1} \beta}{Q_{2}}\right)$, since $\left(\frac{1}{Q_{2}}\right)$ is the average waiting time of heavy drug users at that level of infection. 
Mathematical Modelling on the Effects of Drug Abuse to the Societies The value of $\left(\frac{\sigma}{Q_{1}}\right)$ is the probability that a light user escalates into heavy drug use after first being initiated.The term $\Phi_{1}+\Phi_{2}$ represents the probability that heavy a heavy drug user leaves his class and returns.

The term $\left(\frac{1}{1-\left(\Phi_{1}+\Phi_{2}\right)}\right)$ is the expected number of visits to the $H$ class given that an individual has advanced to $\mathrm{H}$.

The drug-free equilibrium (DFE) is recognized when $L^{*}=0 ; H^{*}=0 ; T^{*}=0$. From the first equation of system (1), we obtain $S^{*}=\left(\frac{\Pi}{\mu}\right)$. Therefore, the drug free steady state isgiven by

$$
\varepsilon_{0}\left(S^{*}, L^{*}, H^{*}, T^{*}\right)=\left(\frac{\Pi}{\mu}, 0,0,0\right) .
$$

When there is no substance abuse in the population, the susceptible population agrees to the total population with a constant steady state $N^{*}=S^{*}=\left(\frac{\Pi}{\mu}\right)$ This steady state is assumed to have a constant inflow from the non-core population at a rate $\Pi$ and an outflow $\mu$, such that an individual is expected to remain in the $\mathrm{S}$ compartment an average of $\mu^{-1}$ years.

Theorem 3.1.The disease-free equilibrium $\mathbb{E}_{0}$ of the system (3) is globally asymptotically stable if $R_{0} \geq 0$ and unstable if $R_{0}<0$.

Proof: Let $V(L, H, T)=a L+b H+c T$ be a candidate Lyapunov function for some nonnegative values of $a, b$ and $c$.

The time derivative of $\mathrm{V}$ is given by

$$
\frac{d V}{d t}=a \frac{d L}{d t}+b \frac{d H}{d t}+c \frac{d T}{d t}
$$

We can now choose the coefficients such that

$$
\begin{gathered}
a=Q_{1} Q_{2}\left(1-\Phi_{1}\right), \\
b=Q_{3}\left(\beta \eta_{1}+\gamma_{2}\right)+\rho \beta \eta_{2}, \\
c=\beta \eta_{2} Q_{2}+\gamma_{3}\left(\beta \eta_{1}+\gamma_{2}\right) .
\end{gathered}
$$

Using these coefficients, the time derivative of the Lyapunov function can be expressed as

$$
\frac{d V}{d t}=Q_{2} Q_{3} Q_{1}\left[1-\left(\Phi_{1}+\Phi_{2}\right)\right]\left(R_{0}-1\right) L
$$

\subsection{Drug persistent equilibrium (DPE)}

Is the time-invariant state with the infection (drug use) present in the population.

The model system (3) has Drug persistent steady state

where

$$
\mathbb{E}_{1}=\left(S^{*}, L^{*}, H^{*}, T^{*}\right)
$$

$$
\begin{array}{r}
S^{*}=\frac{\pi\left[Q_{3} Q_{2}\left(1-\Phi_{1}\right)+Q_{2} \sigma+\rho \sigma\right]}{Q_{3} Q_{2}\left(R_{0}-1\right) Q_{1}\left[1-\left(\Phi_{1}+\Phi_{2}\right)\right]+\mu\left[Q_{3} Q_{2}\left(1-\Phi_{1}\right)+Q_{2} \sigma+\rho \sigma\right]^{\prime}} \\
H^{*}=\frac{\sigma Q_{2} S^{*}\left(R_{0}-1\right)}{\left[Q_{3} Q_{2}\left(1-\Phi_{1}\right)+Q_{2} \sigma+\rho \sigma\right]^{\prime}}, \\
L^{*}=\frac{Q_{2}\left(1-\Phi_{1}\right) S^{*}\left(R_{0}-1\right)}{\left[Q_{3} Q_{2}\left(1-\Phi_{1}\right)+Q_{2} \sigma+\rho \sigma\right]},
\end{array}
$$


Fikiri Lucas Matonyaand Dmitry Kuznetsov

$$
T^{*}=\frac{\sigma S^{*}\left(R_{0}-1\right)}{\left[Q_{3} Q_{2}\left(1-\Phi_{1}\right)+Q_{2} \sigma+\rho \sigma\right]}
$$

Using the overhead expressions, we can have a statement of principle that can be demonstrated and this leads us to the following result;

Theorem 3.2. System (3) has a unique drug-persistent equilibrium

$$
\mathbb{E}_{1}=\left(S^{*}, L^{*}, H^{*}, T^{*}\right)
$$

When the center various theory described in to find the local stability of the endemic steady state. Let us consider the system of equations (1) with the bifurcation parameter $\Phi$ such that

$$
\begin{gathered}
\frac{d x}{d t}=f(x, \Phi), f: \mathbb{R}^{4} x \mathbb{R} \rightarrow \mathbb{R} \\
\text { and } f \in c^{2}\left(\mathbb{R}^{4} x \mathbb{R}\right)
\end{gathered}
$$

Clearly, if 0 is the steady state of the system (3), then $f(0, \Phi)=0$ for all $\Phi$. Let the linearization matrix A

$$
\boldsymbol{A}=D_{X} f(0,0) \text {. }
$$

Have left eigenvector denoted by $y$ and the right eigenvector denoted by $\mathrm{V}$. Then the local dynamics of the model around 0 is totally governed by $\mathbf{a}$ and $\mathbf{b}$, where

$$
\begin{gathered}
a=\sum_{k, i, j=1} y_{k} v_{i} v_{j} \frac{\partial^{2} f_{k}}{\partial x_{i} \partial x_{j}}(0,0) \\
b=\sum_{k, i, j=1} y_{k} v_{i} \frac{\partial^{2} f_{k}}{\partial x_{i} \partial \Phi}(0,0)
\end{gathered}
$$

Let us now define the terms $(S, L, H, T)$ in (1) as $\left(x_{1}, x_{2}, x_{3}, x_{4}\right)$.

We linearize the system of equations (1) at the drugfree equilibrium and with the bifurcation parameter $\Phi$ to obtain

$$
J=\left(\begin{array}{cccc}
-\mu & -\beta^{*} & -\beta^{*} \eta_{1} & -\beta^{*} \eta_{2} \\
0 & \beta^{*}-Q_{1} & \beta^{*} \eta_{1}+\gamma_{2} & \beta^{*} \eta_{2} \\
0 & \sigma & -Q_{2} & \gamma_{3} \\
0 & 0 & \rho & -Q_{3}
\end{array}\right)
$$

Where the parameter $\beta^{*}$ is given by

$$
\Phi=\beta^{*}=\frac{Q_{1} Q_{2} Q_{3}\left[1-\left(\Phi_{1}+\Phi_{2}\right)\right]}{\left[Q_{2} Q_{3}\left(1-\Phi_{1}\right)+\eta_{\sigma} Q_{3}+\eta_{2} \rho \sigma\right]}
$$

We evaluate the left $y$, right $\mathrm{v}$, eigenvectors of the matrix (9), the mixed derivatives of the model system. We then substitute the values in (7) to obtain

$$
\begin{gathered}
a=\frac{2 \beta^{*} \mu y_{2}}{\pi}\left[v_{2} v_{4}\left(1+\eta_{2}\right)+v_{2} v_{3}\left(1+\eta_{1}+v_{3} v_{4}\left(\eta_{1}+\eta_{2}\right)\right]\right. \\
b=\mu Q_{2} Q_{3}\left(1-\Phi_{1}\right)\left[Q_{2} Q_{3}\left(1-\Phi_{1}\right)+\eta_{1} \sigma Q_{3}+\eta_{2} \sigma \rho\right]
\end{gathered}
$$

We observe that $a<0$ and $b>0$. Thus the drug persistent steady state is locally asymptotically stable when $R_{0}>1$ but only if $R_{0}$ is close to 1 .

\subsection{Sensitivity analysis}

In this segment we carry out sensitivity analysis to find out the input of some vital parameters to the dynamics of the model and determine extraordinary behavior of the model if such input parameters are varied. [10] This helps us to allot qualitatively and quantitatively, the variation in the output of the mathematical model to different input 
Mathematical Modelling on the Effects of Drug Abuse to the Societies

variables. Various methods such as differential sensitivity analysis, one at a time sensitivity measures, factorial design, sensitivity index, importance factors, and subjective sensitivity analysis [11], have been used to carry out sensitivity analysis. Of all these methods, subjective sensitivity analysis is the only qualitative method and it relies on the view of experienced investigators [11]. In this thesis, we use sensitivity indices by determining the relative change in the reproduction number when a model parameter change. We use the normalized forward sensitivity index of the reproduction number to the model parameters described in [11]. This is defined as the relative change in the variable $R_{0}$ to the relative change in the parameter. We let $R_{0}$ be a differentiable function of each of the parameters.

Definition 3.1. Let Definition 3.1. Let $R_{0}$ be a differentiable function on the parameter $\varrho$, then the normalized forward sensitivity index of $R_{0}$ at ${ }_{\mathbf{L}}$ is defined as be a differentiable function on the parameter $\mathbf{Q}$, then the normalized forward sensitivity index of $R_{0}$ at $Q$ is defined as

$$
\Upsilon_{\varrho}^{R_{0}}=\frac{\partial R_{0}}{\partial \varrho} \times \frac{\varrho}{R_{0}} .
$$

The quotient $\frac{\mathrm{e}}{R_{0}}$,is introduced to normalize the coefficient by removing the effect of units. In addition, this calculation is based on the assumption that higher order partial derivatives are negligible and that no correlation exists between input parameters [11]. We now use the explicit formula for $R_{0}$ in (10a) and the definition (10b), to evaluate the analytical expressions for sensitivity of $R_{0}$, to the parameters described. For example, the sensitivity index of $R_{0}$ to the contact rate $\beta$ is given as

$$
\gamma_{\beta}^{R_{0}}=\frac{\partial R_{0}}{\partial \beta} \times \frac{\beta}{R_{0}}=1 .
$$

Clearly, the normalized forward sensitivity index (NFSI) with respect to $\beta$ does not depend on any of the parameter values. However, we note that increasing the contact is directly proportional to $R_{0}$ and therefore increasing $\beta$ by $2 \%$ increases $R_{0}$ by the same percentage. Increase in the contact rate consequently increases prevalence of substance abuse, see Figure 3.6. The indices of relative initiation rates of heavy users and drugs users on treatment have obvious structures respectively as

$$
\begin{aligned}
& Y_{\eta_{1}}^{R_{0}}=1-\frac{\left[Q_{2} Q_{3}\left(1-\Phi_{1}\right)+\eta_{2} \sigma \rho\right]}{\left[Q_{2} Q_{3}\left(1-\Phi_{1}\right)+\eta_{1} \sigma Q_{3}+\eta_{2} \sigma \rho\right]} \\
& Y_{\eta_{2}}^{R_{0}}=1-\frac{\left[Q_{2} Q_{3}\left(1-\Phi_{1}\right)+\eta_{1} \sigma Q_{3}\right]}{\left[Q_{2} Q_{3}\left(1-\Phi_{1}\right)+\eta_{1} \sigma Q_{3}+\eta_{2} \sigma \rho\right]}
\end{aligned}
$$

The rest of the expressions of normalized forward sensitivity indices are complex. Therefore, we evaluate the indices of all the rest of the parameters at the nominal values and give the corresponding results. The sign of the calculated value indicates whether, the parameter increases the reproduction number (positive sign) and consequently the prevalence, or reduces the reproduction number (negative sign) and hence the prevalence. 


\section{Fikiri Lucas Matonyaand Dmitry Kuznetsov}

Based on the nominal parameter values, the sensitivity indexes of parameters $\beta$, $\eta_{1}, \eta_{2}, \sigma \sigma$ and $\gamma_{3}$ are positive. The magnitude of the NFSIs of these parameters indicates that, $R_{0}$ is more sensitive to changes in $\beta$ (NFSI=1). The parameters $\mu, \gamma_{1}, \gamma_{2}, \rho, \delta_{1}, \delta_{2}$ and $k$ have negative NFSIs and therefore increasing such parameters reduces $R_{0}$. Of all such parameters $\gamma_{1}$ with $|\mathrm{NFSI}|=0.56$ is the most sensitive. [12] The use and abuse of substances would continue to spread among commercial drivers if the drug abuse number is less than one and the abuse of drugs would die out of the system if the abuse number is greater than one.

\section{Numerical simulation}

\subsection{Parameter estimation}

Natural mortality rate $\boldsymbol{\mu}$ : The average life expectancy of Tanzanian is 50 years [1] and the corresponding mortality rate is thus 0.02 per year.

Drug related removal rates $\delta_{1}$ and $\delta_{2}$ : The time people engage in high risk behaviour under the influence of drugs is not known, and probably varies between populations [41]. Therefore, precise estimation of mortality/removal rates related to substance abuse remains a relatively daunting task. In [13], mortality rates related to drugs among crankcocaine users and injecting drug users are 0.018 and 0.008 respectively. According to [13], a man who stops smoking at 35 years of age can increase his life expectancy by 5 years. We assume a reduction in life expectancy of $14 \%$ due to general substance abuse. With the corresponding $\mu=0.02 y r^{-1}$, the mortality rate of heavy users related to substance abuse $\delta 1$ is $0.0028 y^{-1}$,. Noting that treatment improves the quality of life, we assume that treatment reduces mortality rate related to drugs by at-least $50 \%$. Thus, we choose $\delta_{2}=0.0014 y r^{-1}$,

Progression rate $\sigma$ : In [17], the progression rate from light use to heavy use is 0.47 . This incorporates progressions from light use to moderate use and then escalation heavy use. On the other hand, the value used was 0.024 [14] and between $(0.003,0.004)$ in [15]specific to methamphetamine users. In three major cities in Tanzania 54\% of arrestees who tested positive for cannabis had used the drug in the past 30 days. We may assume that all these arrestees had been heavy cannabis users. We can therefore choose a general progression rate of 0.56 per annum.

Recruitment into rehabilitation $\rho$ : Individuals to be put under rehabilitation must meet the Diagnostic and Statistical Manual of Mental Disorder-Fourth Edition (DSM- IV) criteria for substance abuse or substance dependence. in the survey results from at least $80 \%$ specialist substance abuse treatment centers, it was observed that the proportion of patients under the age of 20 years from January 1997 to December 2001, increased from $5.5 \%$ to $24.1 \%$ and from $7.0 \%$ to $22.0 \%$ in Cape Town respectively. In Gauteng, the proportion increase from $9.9 \%$ to $23.4 \%$ since the between 1998 - 2001 [16]The observed treatment demand for cannabis users is $17 \%$ [17].There was an observed increase in cannabis use in the first two quarters of 2008 in Africa, and including alcohol, the observed treatment demand for cannabis accounted for $23.5 \%$ of all substance abuse [18]According to [19]treatment of drug users accounted for $20 \%$ of all Medicare hospitalizations. We assume this value corresponds to the treatment demand. The upper 
Mathematical Modelling on the Effects of Drug Abuse to the Societies

limit of the treatment demand $(0.009,0.3)$ for methamphetamine users corresponds to $30 \%$ treatment rate. In this paper, we choose the average treatment demand of $22.3 \%$ with the corresponding treatment rate of 0.223 .

\begin{tabular}{|l|l|l|l|l|}
\hline Parameter & Nominal value & Range & Source & $\begin{array}{l}\text { Sensitivity } \\
\text { Index }\end{array}$ \\
\hline$B$ & 0.105 & $0.10-0.21$ & {$[15]$} & 1 \\
\hline$\eta_{1}$ & 0.8 & $0-1$ & Estimated & +0.39 \\
\hline$\eta_{2}$ & 0.6 & $0-1$ & Estimated & +0.14 \\
\hline$\Pi$ & 0.040 & $0.028-0.080$ & Estimated & \\
\hline$\mu$ & 0.020 & $0.019-0.021$ & {$[20]$} & -0.14 \\
\hline$\Sigma$ & 0.560 & $0.400-0.700$ & Estimated & +0.15 \\
\hline$\gamma_{1}$ & 0.200 & $0.10-0.6$ & Estimated & -0.56 \\
\hline$\gamma_{2}$ & 0.20 & $0.20-0.50$ & Estimated & -0.13 \\
\hline$\gamma_{3}$ & 0.25 & $0.10-0.30$ & Estimated & +0.12 \\
\hline$P$ & 0.223 & $0.17-0.30$ & Estimated & -0.20 \\
\hline$\delta_{1}$ & 0.003 & $0.0015-0.0035$ & Estimated & -0.01 \\
\hline$\delta_{2}$ & 0.002 & $0.0013-0.002$ & Estimated & -0.002 \\
\hline$K$ & 0.20 & $0.15-0.50$ & Estimated & -0.22 \\
\hline
\end{tabular}

Table 3.2: Parameter values used in simulations and sensitivity analysis.

To determine the effect of drug abuse to the societies, our numerical simulations for the prevalence of substance abuse for different effective contact rates between drug users and potential users. These results are presented in the figures below.

\section{Prevalence with contact rate}

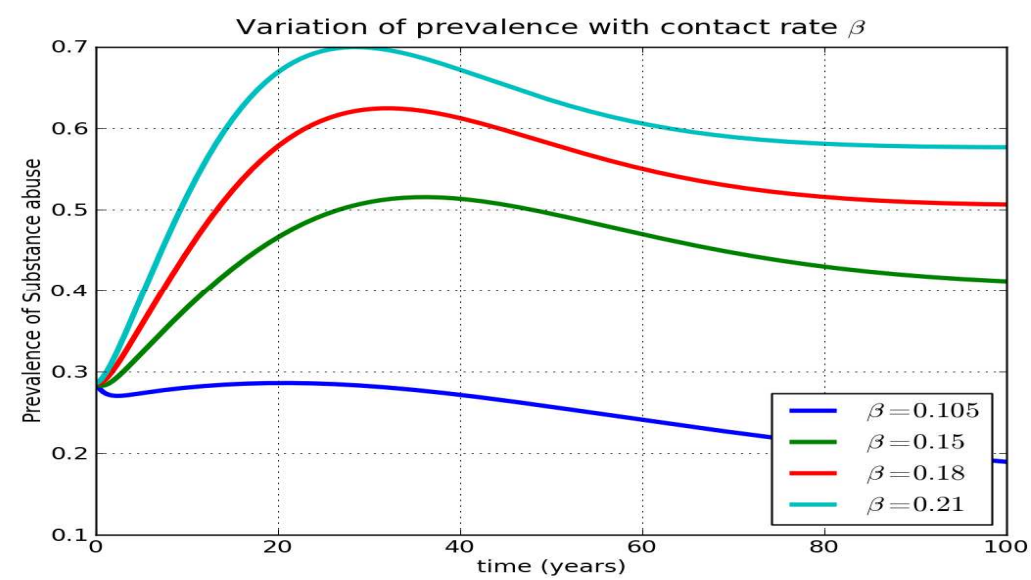

Figure 3.1: Increase in effective contact between drug users and the susceptible population, increases prevalence of substance of substance abuse. Therefore, preventing contact and initiation into drugs greatly reduces the prevalence of substance abuse. 
Fikiri Lucas Matonyaand Dmitry Kuznetsov

Prevalence with rehabilitation

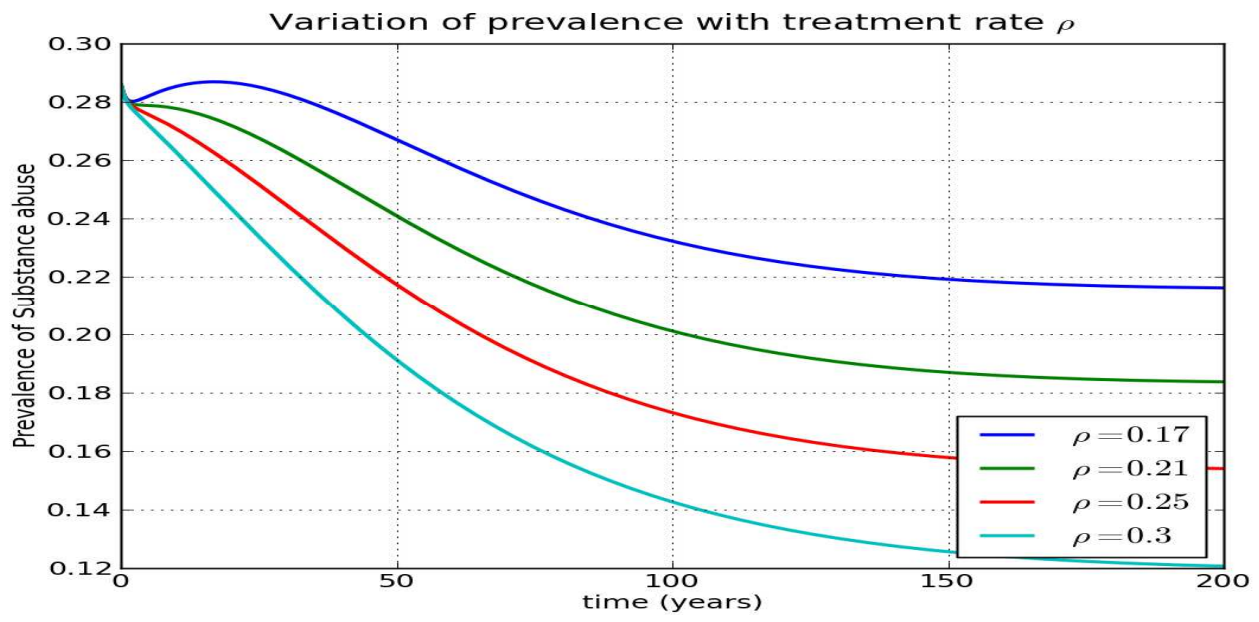

Figure 3.2: Rehabiliationreduces the prevalence of drug epidemics.

Prevalence with re-initiation

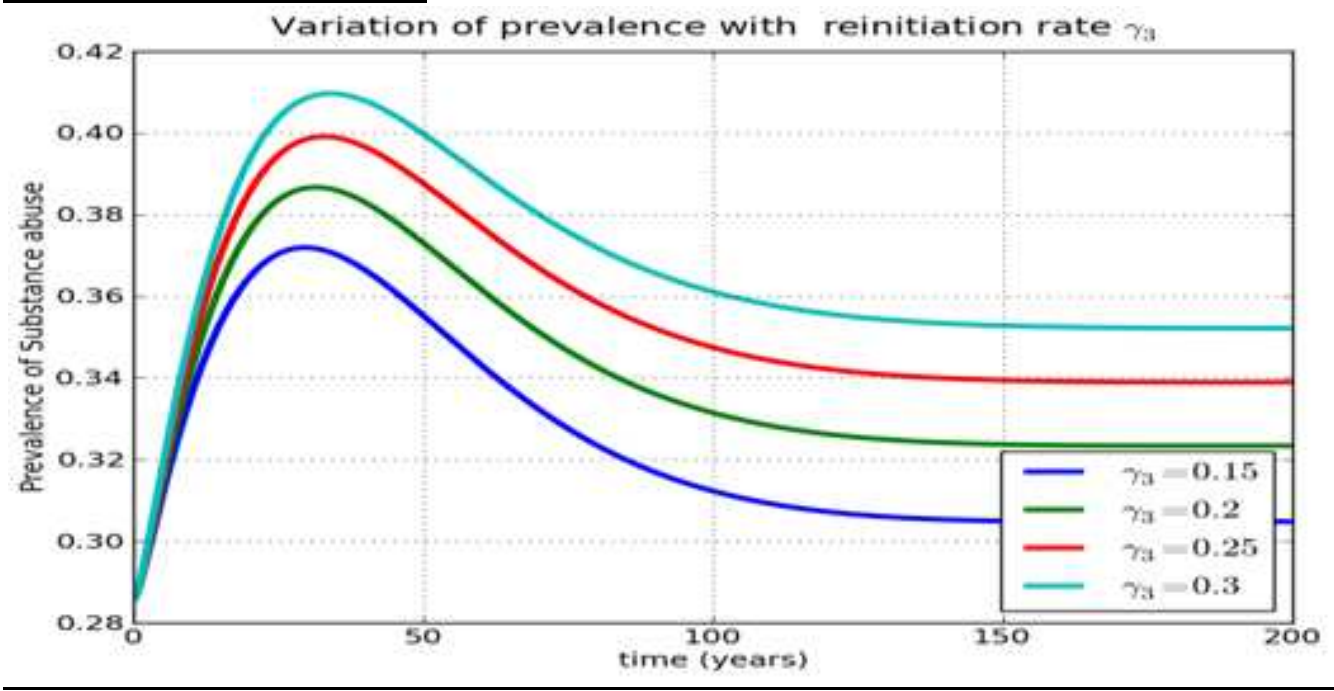


Mathematical Modelling on the Effects of Drug Abuse to the Societies

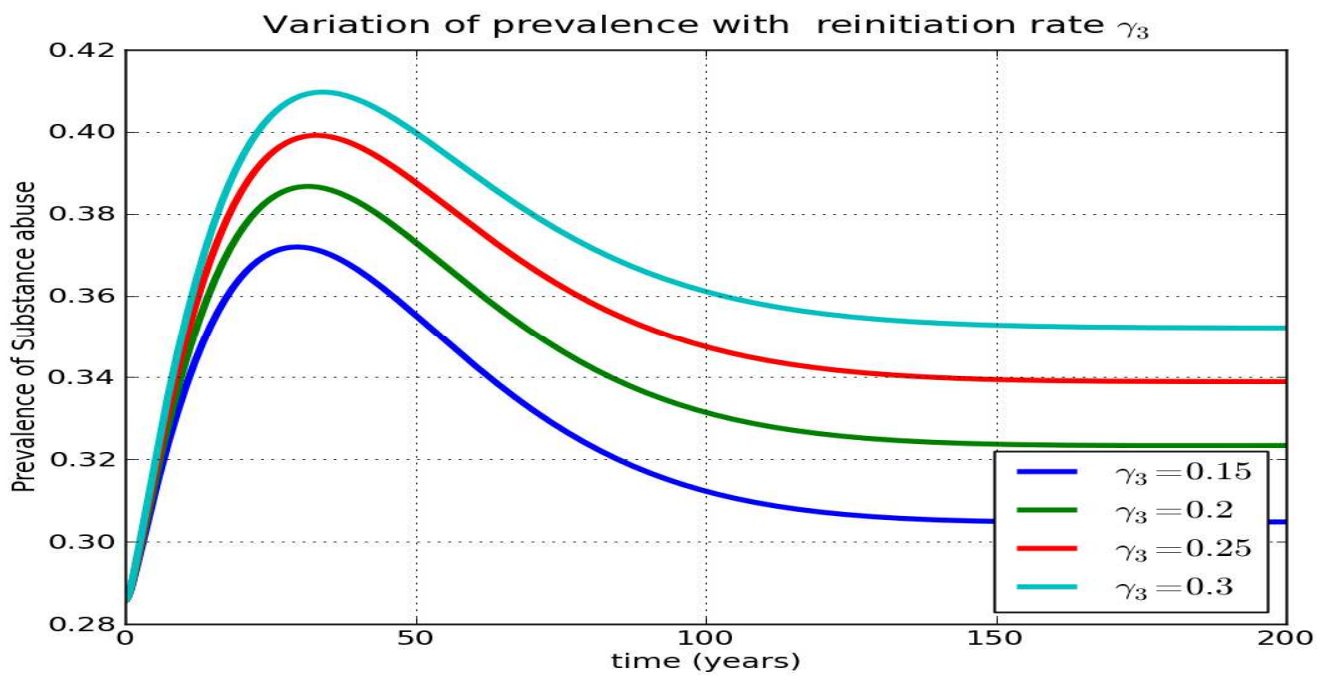

Figure 3.3: Re-initiation increases the prevalence of drug epidemics. Re-initiation/ relapse results in cycling of drug users between heavy use and rehabilitation.

Prevalence with amelioration.

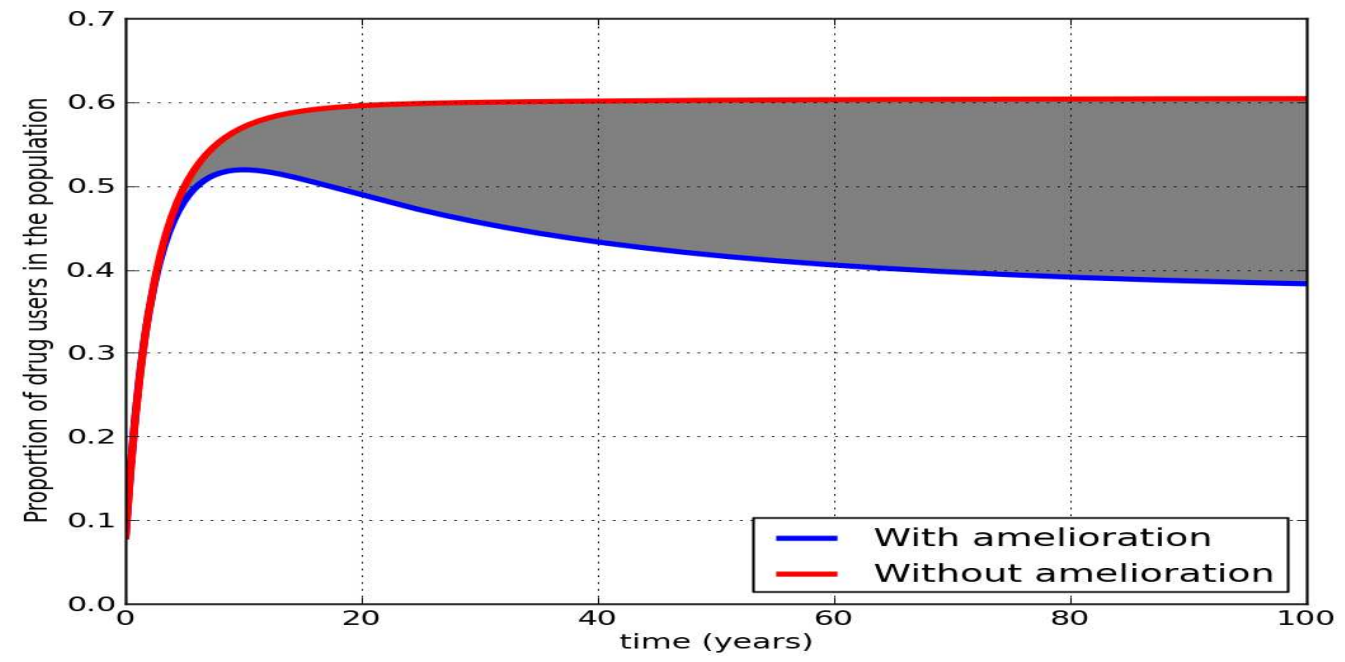

Figure 3.4: Amelioration reduces the prevalence of drug epidemics.Amelioration in presence of quitting for light users, results in reduction in prevalence of substance abuse. However, amelioration in absence of quitting for light users results in cycling of drug users between the heavy use and light use, thus increasing prevalence

\section{Conclusion}

Mathematical analysis of the model with amelioration, indicates that there exists a drug free steady state which is globally stable whenever $R_{0}<1$ and a unique drug persistent 


\section{Fikiri Lucas Matonyaand Dmitry Kuznetsov}

steady state which is globally stable when $R_{0}>1$. The epidemiological interpretation of this being that, substance abuse can be eliminated if the reproduction number is reduced to a value below unity.The importance of model parameter to the prevalence and spread of drug epidemics was investigated using the normalized forward sensitivity indices with respect to the reproduction number. This sensitivity analysis together with the numerical simulations consistently show that prevalence of substance abuse increases with increase in the influence (contact) of drug users on the susceptible population, reinitiation (relapse or reversion) into drug use and amelioration in the absence of quitting for light users. On the other hand, prevalence of substance abuse reduces with increase in recruitment into rehabilitation, and with amelioration in presence of quitting for light users. In this research, we were able to understand the drugs abused, and the key factors that influence the dynamics of substance abuse.

Our recommendation for further research on this topic are as follows.

1. The fact that different age groups have different drug using patterns, it is important to structure the population into different age groups. This may help researchers and policy makers to;

- $\quad$ assess the demographic impact of drugs with significant morbidity and mortality.

- $\quad$ estimate parameters related to drug use patterns from age-specific data.

- $\quad$ design control and intervention programs to protect vulnerable age groups.

2. Data on the numbers of drug users should be collected, for each specific drug accounting for multiple drug usage. This data will be important in looking for synchrony of drug epidemics over the different provinces and possible periodicity in drug use patterns.

All these will help in better understanding of drug use patterns and designing proper control and rehabilitation strategies.

Acknowledgements. The author appreciates the support of Africa Development Bank in the production of the manuscript and covering all the costs, the School of Computation and Communication Science and Engineering of Nelson Mandela African institute of science and technology and the entire team of Annals of Pure and Applied Mathematics Journal (APAM).

Competing interests: We declare that we have no financial or personal relationships which may have inappropriately influenced us in writing this article.

\section{REFERENCES}

1. World Health Organization, Neuroscience of psychoactive substance use and dependence, Addiction, 99(10) (2004) 1361-1362.

2. C.D.H.Parry, Substance Abuse in South Africa: Country Report, no. February, pp. 24-26, 1998.

3. Pacific Institute Series on Forensic Psychology.

4. N.Volkow and T.-K.Li, Volkow ND, Li TK. Drug addiction: the neurobiology of behaviour gone awry, Nat Rev Neurosci, 5 (2005) 963-970.

5. B.Sproule, Handbook of the Medical Consequences of Alcohol and Drug Abuse, 2nd 
Mathematical Modelling on the Effects of Drug Abuse to the Societies edition, Can. J. Hosp. Pharm., 62(3) (2009) 252.

6. T.Rhodes, S.Bivol, O.Scutelniciuc, N.Hunt, S.Bernays and J.Busza, Narrating the social relations of initiating injecting drug use: Transitions in self and society, Int. J. Drug Policy, 22(6) (2011) 445-454.

7. S.Osman, O.D.Makinde and D.M.Theuri, Stability analysis and modelling of listeriosis dynamics in human and animal populations, 14(1) (2018) 115-137.

8. G.Macdonald, Epidemiologic models in studies of vectorborne diseases, Public Health Rep., 76 (1961) 753-64.

9. P.Van Den Driessche and J.Watmough, Reproduction numbers and sub-threshold endemic equilibria for compartmental models of disease transmission, Math. Biosci., 180 (1-2) (2002) 29-48.

10. K.A.Eustace, Mathematical modelling and analysis of the dynamics of cholera, Report no. October, 2018.

11. D.M.Hamby, A review of techniques for parameter sensitivity analysis of environmental models, Environ. Monit. Assess., 32(2) (1994) 135-154.

12. J.K.Kanyaa, S.Osman and M.Wainaina, Mathematical modelling of substance abuse by commercial drivers, Report no. October, 2018.

13. M.Askarian, F.Kouchak, M.Youssef and L.M.Romito, Comparing tobacco use know -ledge, attitudes and practices between engineering students at a public and Islamic Azad university in Shiraz, Iran 2011, Int. J. Prev. Med., 4(10) (2013)1154-1161.

14. C.Rossi, Operational models for epidemics of problematic drug use: The MoverStayer approach to heterogeneity, Socioecon. Plann. Sci., 38(1) (2004) 73-90.

15. F.Nyabadza and L.Coetzee, A systems dynamic model for drug abuse and drugrelated crime in the western cape province of south africa, Comput. Math. Methods Med., 2017 (2017) 4074197.

16. L.Cherian and M.Mboweni, Patterns and prevalence of alcohol use among university of utopia students in South Africa, Mediterr. J. Soc. Sci., 5(20) (2014) 1573-1579.

17. K.Peltzer and S.Ramlagan, Cannabis use trends in South Africa, South African J. Psychiatry, 13(4) (2007)126-131.

18. United Nations Office on Drugs and Crime, World drug report 2009. 2009.

19. W.Summit and S.Development, The social impact, Soc. Dev., Report no. March, pp. 6-12, 1995.

20. D.B. and I.M.Timaeus, Disease and Mortality in Sub-Saharan Africa. 2006. 